\title{
ISI Cancellation Using Blind Equalizer based on DBC Model for MIMO-RFID Reader Reception
}

\author{
Sarun DUANGSUWAN, Sathaporn PROMWONG \\ Dept. of Telecommunication Engineering, Faculty of Engineering, King Mongkut's Institute of Technology Ladkrabang, \\ Chalongkrung Rd., Ladkrabang, Bangkok 10520, Thailand \\ kdsarun@kmitl.ac.th, kpsathap@kmitl.ac.th
}

\begin{abstract}
Considering a dyadic backscatter channel (DBC) model, the previous methods with zero forcing $(Z F)$ and minimum mean squared error (MMSE) are not able to rapidly cancel inter-symbol interference (ISI) because of errors of post-preamble transmission in MIMO-RFID channel. In order to achieve the ISI cancellation, the proposed method is demonstrated to improve the convergence rate without postpreamble by using a constant modulus algorithm (CMA) in this paper. The CMA depends on the steepest descent algorithm function, which is based on the second order statistics (SOS) to estimate the channel characterization. We compare between the conventional method and the proposed method based on simulation and measured data. Furthermore, the multiple tag of post-processing is also considered under the assumption of the maximum likelihood detection. We can confirm that the proposed method is better than the conventional method with faster ISI cancelling and a lower bit error rate (BER) improving up to 12 tags.
\end{abstract}

\section{Keywords}

RFID reader, MIMO channel, DBC model, blind equalizer, ISI cancellation

\section{Introduction}

The RFID system is a wireless communication that utilizes radio-frequency (RF) waves to identify objects. The communication system relies on data transmission between an RFID tag and interrogator or RFID reader, where reading range depends on the RFID reader to emit the RF with an inductive coupling or electromagnetic wave. The communication systems of reader to tag calls that a forwarding, and tag to the reader is a backscattering respectively. Nowadays, there are several used standard frequencies such as $125-134 \mathrm{kHz}$ and $13.56 \mathrm{MHz}$ for short reading range in a near-field communication and $860-960 \mathrm{MHz}$ and $2.45 \mathrm{GHz}$ to $5.8 \mathrm{GHz}$ for a long reading range in far-field communication. On the other hand, the research trends are focusing on the advantage of microwave band at $2.45 \mathrm{GHz}$ that provides a higher data rate of the transmission system for the wireless identification and sensing platform (WISP) [1]. Develop- ing a faster signal processing or higher order modulation has been also focused on such as QPSK and QAM in the passive tag, which can improve poor tag sensitivity and power harvesting of the backscatter modulation [2].

Meanwhile, development of the RFID reader has been focused by using software defined radio (SDR) [3]. With the flexibility and programmability of signal processing, a digital signal processor (DSP) and field programmable gate array (FPGA) are primary devices in digital baseband transceiver [4]. Corresponding to these, the SDR platform has been studied by the interesting introduction in [5] and [6]. The platform provides a universal software radio peripheral (USRP) to investigate the tag reading range and received signal sensitivity, where ten commercial tags and four built-in tags are used. Even though easily configurable, however, the sensitivity of the RFID reader is dependent upon the receiving antenna and propagation delay. The main problem is multipath fading [7]-[11]. Thus, a solution including $N \times L \times M$ DBC model [10], multiple transmit antenna [12]-[13], space-time coding [14], and blind source separation [15] is also described.

In order to seek the pioneering works of the MIMORFID channel processing, [16]-[18] proposed advanced signal processing to resolve the error detection such as linear filtering and linear equalizing. Among well known a postprocessing, maximum ratio combining (MRC) as a solution technique that was presented in [19] is interesting. This method optimizes the signal to noise ratio (SNR) output in each of antenna branches. Unfortunately, the MRC needs to know the a posteriori probability at the receiver. With a set of the posteriori probabilities, it depends on the degree of freedom of the channel state information (CSI). Although the posterior or post-preamble has been described in the works [16] and [20], however, the discussion is still focused on reducing the ISI problem. Generally, there are many works focused on the ZF and MMSE receiver. In the conventional method [16], it is described that these can be optimized for the collision recovery up to $L=2 M$ as a number of tags, where $M$ represents the multiple receiving antennas. Even though it has low complexity, the solution is still limited with a weakness of ISI cancellation in DBC model. In principle, [21] discussed that a communication theoretic approach of the multi-packet reception for the RFID reader can provide a 
blind channel estimation and equalization at the baseband receiver. Corresponding to this discussion, blind equalization has been introduced in [22]. The authors confirmed that the performance of blind equalization is better than non-blind equalization. Additionally, an application of blind equalization can be used to reduce the ISI in a complex channel rapidly. Although the RFID system has been used since 2004, an evaluation of them has not been focused on using the blind equalization to solve the severity of ISI [23].

This paper presents an evaluation of ISI cancellation by using a blind equalizer based on the DBC model for the MIMO-RFID reader reception. In order to resolve a slow convergence rate of the conventional method [16], an approach of ZF and MMSE methods is discussed by utilizing a constant modulus algorithm (CMA), which can mitigate the ISI rapidly. Furthermore, the multiple tag postprocessing is also considered under the assumption of the maximum likelihood detection. For DBC model [10], the channel $N \times M$ was measured by using the vector network analyzer (VNA) HP8510C as a channel sounding, and $L$ was simulated. The experimental results show the performance evaluation between the proposed method and the conventional method with ISI cancelled. Also, the BER performances are discussed.

The remainder of the paper is organized as follows. The signal models are described in Sec. 2 and Sec. 3 focuses on the conventional method and the proposed method of the RFID reader reception. Finally, a description of the experimental results is shown in Sec. 4 and the conclusion is presented in the last section.

\section{Signal Modeling}

This section describes a signal modeling of MIMO in RFID system [10]. The $N \times L \times M$ DBC model described the MIMO system where $N$ denotes the transmitting antennas, $L$ denotes the multiple tag, and $M$ receiving antennas.

The received signal is given by the vector $\mathbf{r} \in \mathrm{C}^{M \times 1}$

$$
\mathbf{r}=\mathbf{H}^{\mathrm{f}} \mathbf{s} \mathbf{H}^{\mathrm{b}} \mathbf{x}+\mathbf{i}+\mathbf{n}
$$

where $\mathbf{H}^{\mathrm{f}}$ is a forwarding channel vector, $\mathbf{H}^{\mathrm{b}}$ is a backscattering channel vector. The vector $\mathbf{x}=$ $\left[x_{1}(t), x_{2}(t), \ldots, x_{N}(t)\right]^{T} \in \mathrm{C}^{N \times 1}$ is a matrix of the transmitted carrier signal in forwarding. In addition, $\mathbf{s}=$ $\left[s_{1}(t), s_{2}(t), \ldots, s_{L}(t)\right]^{T} \in \mathrm{C}^{L \times L}$ as backscattered signal and $\mathbf{n}=\left[n_{1}(t), n_{2}(t), \ldots, n_{M}(t)\right]^{2} \in \mathrm{C}^{M \times M}$ the complex noise vector. Finally, $\mathbf{i}=\left[i_{1}(t), i_{2}(t), \ldots, i_{N}(t)\right]^{T} \in \mathrm{C}^{M \times M}$ denotes a mutual interference of the receiving antennas. Note that $[\cdot]^{T}$ is a matrix transpose.

The respective of the signaling matrix $\mathbf{s}$ is given as a sum of $L$ independent RF tags

$$
\mathbf{r}=\sum_{l=1}^{L} s_{l}(t) \mathbf{H}^{\mathrm{f}} \mathbf{x} \mathbf{H}^{\mathrm{b}}+\mathbf{i}+\mathbf{n} .
$$

Then, a simplicity channel matrix is

$$
\mathbf{r}=\sum_{l=1}^{L} \mathbf{H} s_{l}(t)+\mathbf{i}+\mathbf{n}
$$

where $\mathbf{H}=E\left[\mathbf{H}^{\mathrm{f}} \mathbf{H}^{\mathrm{b}}\right]$ is an expectation of the channel transfer function in the DBC model $\mathbf{H} \in \mathrm{C}^{N \times M}$ and the matrix of the transmitted signal is determined by $\mathbf{x} \in \mathrm{C}^{N \times 1}=1$.

From the channel matrix, the channel power at the $M$ receiving antenna branch, also referred as the instantaneous signal to interference plus noise (SINR), is given by

$$
\gamma=\frac{E[\mathbf{H s}]}{E[\mathbf{i}+\mathbf{n}]}=\sum_{n=1}^{N} \sum_{l=1}^{L} \sum_{m=1}^{M} \frac{\left|h_{m, n}(t) s_{l}(t)\right|^{2}}{\left|i_{n}(t)+n_{m}(t)\right|^{2}}
$$

where, $E[\cdot]$ denotes the expectation operator. Then, $h_{m, n}(t)$ is a channel impulse response of the system model.

The matrix of channel transfer function $\mathbf{H} \in \mathrm{C}^{N \times M}$, is written as

$$
\mathbf{H}=\left[\begin{array}{cccc}
h_{1,1}(t) & h_{1,2}(t) & \cdots & h_{1, n}(t) \\
h_{2,1}(t) & h_{2,2}(t) & \cdots & h_{2, n}(t) \\
\vdots & \vdots & \ddots & \vdots \\
h_{m, 1}(t) & h_{m, 2}(t) & \cdots & h_{m, n}(t)
\end{array}\right]
$$

\section{RFID Reader Reception}

In order to reflect the high demands on technological performance for the RFID reader reception, the advanced signal processing techniques are required. The MIMO system has been described in the works [12]-[13], and the signal modeling was shown in the previous section. In this section, a channel equalizer in the RFID reader reception is modeled.

\subsection{Conventional Method}

The advantages of MIMO in RFID system are more coverage areas of accurate tracking, identifications, since it can significantly increase the throughput. Consequently, the usage of the multiple antennas increased a reliability of the transmission system. With the advent of the temporal filtering, a channel equalizer can be proposed for decoding tags in the presence of multipath environment and noise enhancement. It has become an integral part of the RFID signal processing design.

The channel equalizer of conventional method [16] is based on perfect channel state information (CSI) for their simplicity, but they are not optimum in a maximum likelihood sense. Although they are not optimal, the ZF and MMSE receiver satisfy an alternative criterion, i.e., it minimize the BER and recover the multiple-tag detection.

Figure 2 shows the conventional method of the RFID reader receiver with MIMO-linear equalizer, where signal modeling of an output post-equalization is given by

$$
\mathbf{y}=\mathbf{G r}
$$




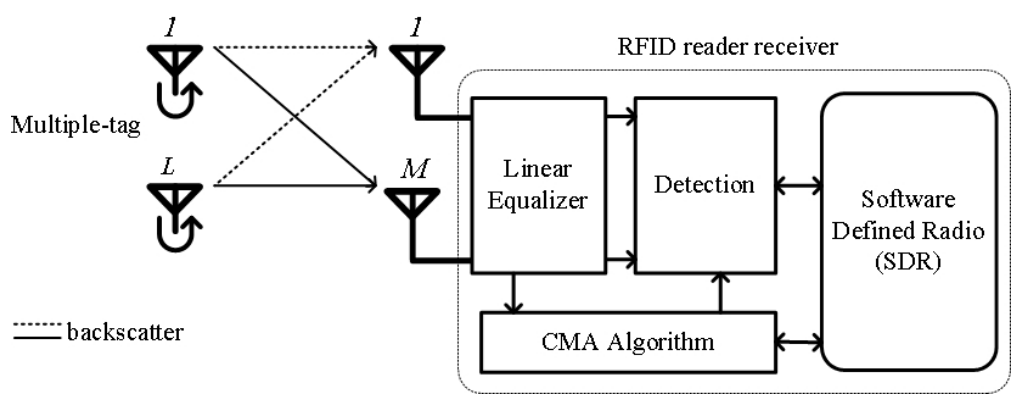

Fig. 1. System model of the RFID reader receiver for the multiple tag detection.

where $\mathbf{G}=\left[G_{\mathrm{zf}, \mathrm{mmse}}^{1}, G_{\mathrm{zf} \text {,mmse }}^{2}, \ldots, G_{\mathrm{zf}, \mathrm{mmse}}^{M}\right]^{T} \in \mathrm{C}^{1 \times M}$ is the matrix of linear equalizer as $\mathrm{ZF}$ and MMSE criterion. The matrix $\mathbf{r}=\left[r_{1}(t), r_{2}(t), \ldots, r_{M}(t)\right]^{T} \in \mathrm{C}^{1 \times M}$ denotes the matrix of the received signal and the matrix $\mathbf{y}=$ $\left[y_{1}(t), y_{2}(t), \ldots, y_{L}(t)\right]^{T} \in \mathrm{C}^{L \times L}$ is the output of the postequalization.

The respective of signaling matrix for $\mathbf{y}$ independent tags is modeled as a sum of the output post-equalization as

$$
y_{1}(t), y_{2}(t), \ldots, y_{L}(t)=\sum_{m=1}^{M} G_{\mathrm{zf}, \mathrm{mmse}}^{m} r_{m}(t) .
$$

In substitution (6), the conventional method that is known $\mathbf{r}$ by using post-preamble, which assumes perfect CSI at the receiver. In noise free case,

$$
\begin{gathered}
\mathbf{y}=\mathbf{G H s}+\mathbf{G n}, \\
\mathbf{y}=\mathbf{G H s},
\end{gathered}
$$

where the output is unknown. It can be estimated by using Moore-pseudo inverse of the channel as $\mathbf{G}=\left(\mathbf{H H}^{H}\right)^{-1}$ and then

$$
\mathbf{y}=\left(\mathbf{H H}^{H}\right)^{-1} \mathbf{H}^{H} \mathbf{s} .
$$

Likewise, in the noise case, the substitution (10) has been a problem by the noise enhancement. This problem is able to be solved instead as $\mathbf{G}=\left(\mathbf{H H}^{H}+\mathbf{n}\right)^{-1}$, and then

$$
\begin{gathered}
\mathbf{y}=\left(\mathbf{H} \mathbf{H}^{H}+\mathbf{n}\right)^{-1} \mathbf{H}^{H} \mathbf{s}+\left(\mathbf{H} \mathbf{H}^{H}+\mathbf{n}\right)^{-1} \mathbf{n}, \\
\mathbf{y}=\left(\mathbf{H H}^{H}+\sigma^{2} \mathbf{I}\right)^{-1} \mathbf{H}^{H} \mathbf{s},
\end{gathered}
$$

where $\sigma^{2}=E\left[\mathbf{n n}^{H}\right]$ denotes its noise variance, $[\cdot]^{H}$ represents a Hermitian transposition and $\mathbf{I}$ is an $M \times M$ identity matrix of the channel equalizer.

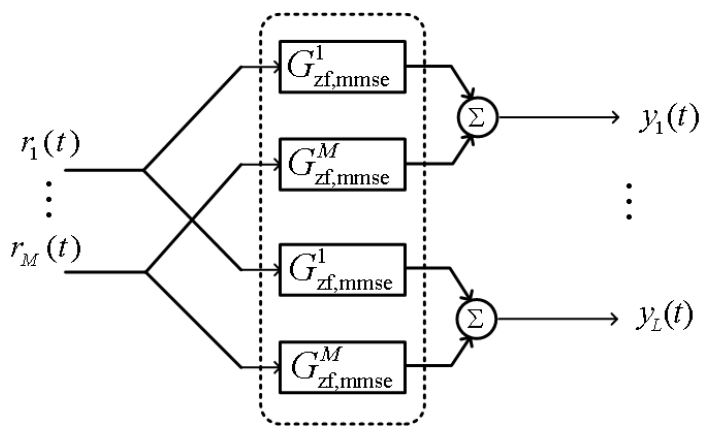

Fig. 2. The conventional method of in the RFID reader receiver with MIMO-linear equalizer [16].

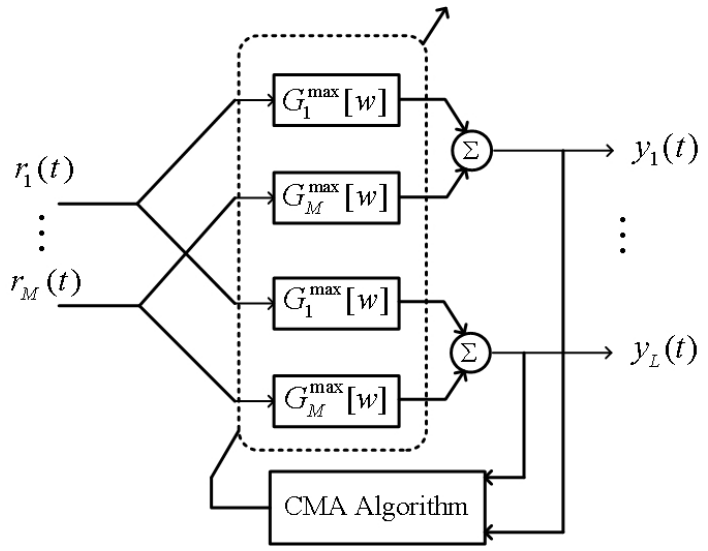

Fig. 3. The proposed method of in the RFID reader receiver with MIMO-blind equalizer.

Thus, the substitution (10) and (12), as well-known, is the term of $\mathrm{ZF}$ and MMSE criterion, respectively.

For simplicity, the performance of the channel equalizer is considered with SNR output as given by

$$
\hat{\gamma}=\frac{E[\mathbf{y}]}{E[\mathbf{n}]}=\sum_{l=1}^{L} \sum_{m=1}^{M} \frac{\left|y_{l}(t)\right|^{2}}{\left|n_{m}(t)\right|^{2}}
$$

and thus asymptotically average BER is given as

$$
P_{\mathrm{b}}=Q(\sqrt{\hat{\gamma}})
$$

where $Q(\cdot)$ denotes the $Q$-function of the error probability.

Unfortunately, the RFID reader receiver-based ZF and MMSE are to raise a very large $\mathbf{H} \mathbf{H}^{H}$, the postpreamble will be increased, and $\hat{\gamma}$ will be reduced.

\subsection{Proposed Method}

Both ZF and MMSE of the conventional method are limited at the detecting up to $L=2 M$ as number of tags. The aim of the proposed method is to achieve the detection of upper 8 tags, where $M=4$ is satisfied. Thus, the system model in Fig. 3 has been proposed by using the CMA algorithm in this paper. Figure 3 shows a structure of ISI cancellation, where $w$ denotes weight coefficient without post-preamble at the receiver.

As a first step, the CMA algorithm is performed with SOS estimation, which has the cost function as 


$$
J_{w}=E\left[|\mathbf{y}|^{2}-\Delta\right]^{2}
$$

where $\Delta$ denotes a real positive constant is calculated by $\Delta=\frac{E\left[|s(t)|^{4}\right]}{E\left[|s(t)|^{2}\right]}$ and a stochastic gradient descent (SGD) algorithm as

$$
w^{(k+1)}=w^{(k)}-\mu \varepsilon \cdot J_{w}
$$

where $\mu$ denotes a small step size parameter of CMA algorithm and $\varepsilon$ represents the estimate of error function using CMA criterion,

$$
\varepsilon=s(t)\left(\Delta-|s(t)|^{2}\right)
$$

In order to define smaller step size parameter of CMA algorithm properly, a sufficient condition is inspired by [24],

$$
0<\mu \leq \frac{2}{3 \operatorname{tr}\left[\mathbf{R}_{\mathrm{ss}}\right]}
$$

where $\operatorname{tr}\left[\mathbf{R}_{\mathrm{ss}}\right]$ is the trace of the backscatter signal $s(t)$ autocorrelation matrix.

As the second step, the multiple-tag is detected under the assumption of the maximum likelihood estimation.

$$
G_{M}^{\max }[w]=\arg \max _{\mathbf{s}} f[\mathbf{s} \mid \mathbf{y}]
$$

where, $f(\mathbf{s} \mid \mathbf{y})$ denotes the likelihood function, then

$$
\begin{aligned}
f[\mathbf{s} \mid \mathbf{y}] & =f\left[s_{1}(t), s_{2}(t), \ldots, s_{L}(t) \mid y_{1}(t), y_{2}(t), \ldots, y_{L}(t)\right] \\
& =\prod_{l=1}^{L} \frac{1}{\sqrt{2 \pi \sigma^{2}}} \exp \left(-\frac{1}{2 \sigma^{2}} \sum_{l=1}^{L} \sum_{m=1}^{M}\left\|s_{l}(t)-w_{m}^{(k+1)} y_{l}(t)\right\|^{2}\right)
\end{aligned}
$$

where $\|\cdot\|$ is the 2-norm of the vector $\mathbf{s}$ and $\mathbf{y}$.

Since the maximization of the $f(\mathbf{s} \mid \mathbf{y})$ is equivalent to the minimization of $\|\mathbf{s}-\mathbf{w y}\|^{2}$, the maximum likelihood searches for the candidate of $s_{L}(t)$ that minimizes the $\log$ likelihood function $\log f(\mathbf{s} \mid \mathbf{y})$ defined as

$$
\begin{aligned}
\log f[\mathbf{s} \mid \mathbf{y}] & =-L \log \sqrt{2 \pi \sigma^{2}} \\
& \left.-\frac{1}{2 \sigma^{2}} \sum_{l=1}^{L} \sum_{m=1}^{M}\left\|s_{l}(t)-w_{m}^{(k+1)} y_{l}(t)\right\|^{2}\right) .
\end{aligned}
$$

Thus, substitution (7) is reconsidered by

$$
y_{1}(t), y_{2}(t), \ldots, y_{L}(t)=\sum_{m=1}^{M} G_{m}^{\max }[w] r_{m}(t)
$$

\section{Experimental Results}

\subsection{Channel Measurement}

Herein, the $N \times M$ channel sounding was carried out by using vector network analyzer (VNA) HP8510C and calibration kits, conducted in a corridor area on the $3^{\text {th }}$ floor of the building, Faculty of Engineering, King Mongkut's Institute of Technology Ladkrabang (KMITL). Fig. 4 shows the measurement floor plan in the top view, where the surrounding environment is a reinforced concrete, window, classroom and the laboratory room. The preparation of the Tx antenna and $\mathrm{Rx}$ antenna consist of a 4 sleeve monopole antenna about $3 \mathrm{dBi}$ per element, connected with 4-way power divider and combiner. The port-1 VNA is connected with Tx antenna and port- 2 connected with Rx-antenna, the calibration methods of the MIMO elements are based on short open load thru (SOLT) technique. Fig. 5 shows the measurement site of the side view, where the height of Tx and Rx antenna was $1.5 \mathrm{~m}$, the distance from the Tx antenna to Rx antenna was $2 \mathrm{~m}$, while the antenna spacing was $0.06 \mathrm{~m}$ (half-wavelength at $2.45 \mathrm{GHz}$ ). The channels were measured for all $4 \times 4$ pairs with VNA as a channel transfer function. The measurement band was from 2.4 to $2.5 \mathrm{GHz}$ (bandwidth $=100 \mathrm{MHz}$ ), and we obtained 801 numbers of frequency points as a sweeping time approximated $7 \mathrm{~ms}$ intervals. We note that the measurement campaign was conducted while no one was in the environment to ensure statistically stationary propagation.

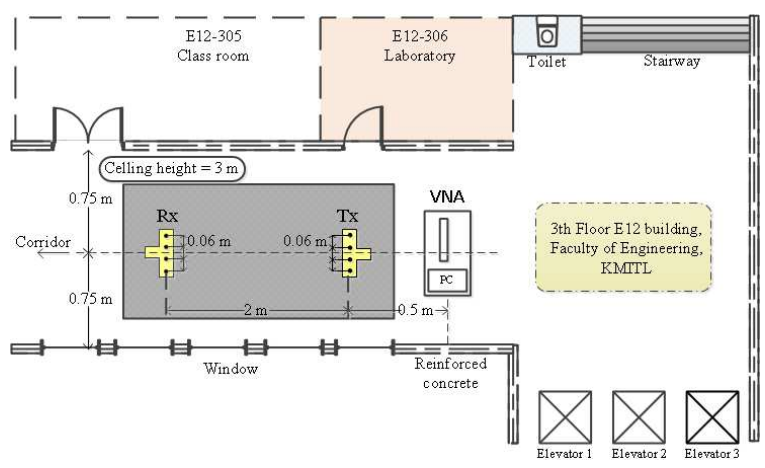

Fig. 4. Measurement floor plan (top view).

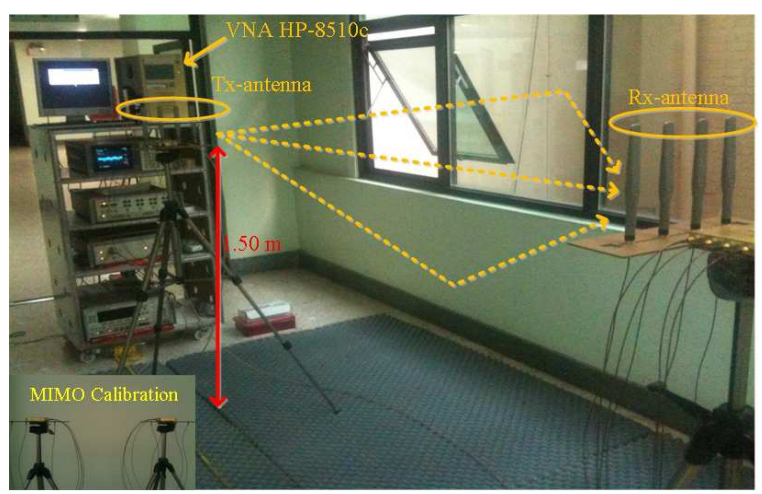

Fig. 5. Measurement site (side view). 


\subsection{Data Processing}

To investigate the ISI cancellation and BER performance, data processing is conducted by using computer simulation and measured channel data. The tag was subject to simulations of the multiple backscatters by assuming the parameters listed in Tab. 1. Some parameters are inspired by [14], such as data rate and QPSK modulation scheme. Because the QPSK is being focused on the passive tag, therefore, the Rx signal processing can be supported by using CMA algorithm as well, which used the step size of 0.01 following [24]. In order to evaluate the ISI severity from the measured channel data, we performed 1000 iterations of the convergence rates. The criterion of the ISI cancelled by using blind equalizer was perfectly optimized as the maximum $10 \mathrm{~dB}$ and $-10 \mathrm{~dB}$ minimum of the threshold and BER satisfied $10^{-4}$.

Figure 6 shows the flow chart of the experimental schemes in this paper. Firstly, we assumed the number of the simulation backscattered $L$ tags through the measured channel data. The channel was done by $N \times M$ indoor under test. The snapshots of the measured channel data were logged as 801 points by the VNA equipment. Secondly, the simulation studies of the conventional method and the proposed method are conducted. In this simulation, a four tap finite impulse response (FIR) of the ZF and MMSE channel inversion were used. In order to estimate the measured channel, the conventional method estimates the channel via the postpreamble, 12 bits of the tag response. The proposed method estimates the channel via the second order statistics of the received signal without postpreamble bits. For the proposed method, all elements of the channel coefficient were weighted by approaching the cost function of the CMA algorithm. In order to cancel the severe ISI, thirdly, we investigated the convergence rate of the ISI cancelled between the conventional method and the proposed method. To this end, we also simulated the BER performance of the multiple-tag detection backscattering link transmission via the measured channel data.

Figure 7 shows the ISI convergence rate between the conventional method and the proposed method. In Fig. 7 (a) as the ZF-CMA with $M=1$ and $L=2$, we note that the proposed method has faster convergence speed and lower ISI (dB) floor than the conventional method [16]. It is observed that the performance of the ZF-CMA is able to reduce ISI as from $15.3 \mathrm{~dB}$ to $-3.3 \mathrm{~dB}$ until stable at $k=300$. Likewise, the MMSE-CMA with $M=1$ and $L=2$ that optimizes the convergence speed is also able to cancel ISI as from $15.3 \mathrm{~dB}$ to $-5.1 \mathrm{~dB}$ until stable at $k=200$ in Fig. 7 (b). Then, we point out the case of $M=2$ and $L=8$. In Fig. 7 (c) ZF-CMA and Fig. 7 (d) MMSE-CMA, note that the ISI maximizes as average $17.6 \mathrm{~dB}$ due to the increasing $L$.

\begin{tabular}{c|c}
\hline No. of Tags $L$ & 12 \\
\hline Data rate for each tag & $640 \mathrm{kbps}[14]$ \\
\hline Modulation scheme & QPSK [14] \\
\hline Data length & 128 symbols \\
\hline Frame duration & $10 \mathrm{~ms}$ \\
\hline Rx signal processing & ZF-CMA, and MMSE-CMA \\
\hline Step size $\mu$ & $0.01[24]$ \\
\hline No. of iterations $k$ & 1000 \\
\hline $\begin{array}{c}\text { Maximum and minimum } \\
\text { threshold of ISI canceling }\end{array}$ & $10 \mathrm{~dB}$ and $-10 \mathrm{~dB}$ \\
\hline BER threshold & $\leq 10^{-4}$ \\
\hline
\end{tabular}

Tab. 1. Simulation parameters.

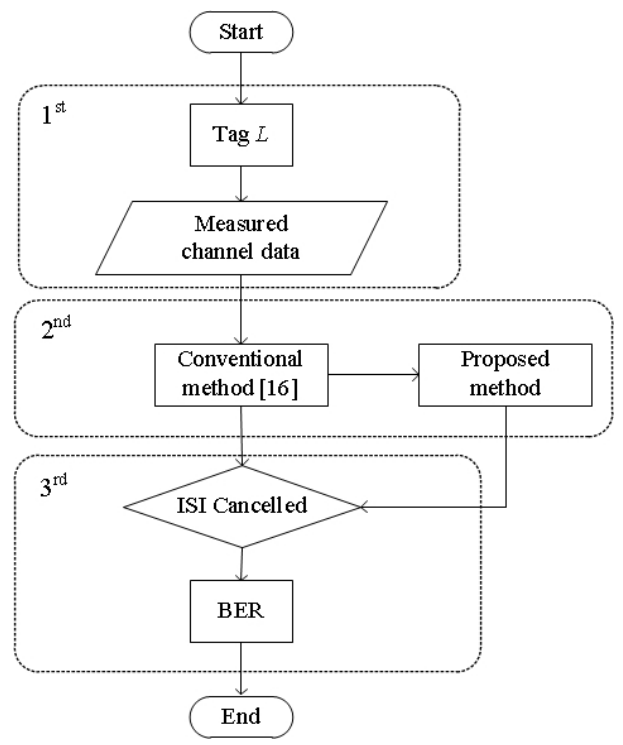

Fig. 6. Flow chart of the experimental schemes.

It is also clear that the proposed method has faster convergence speed and lower ISI (dB) than the conventional method. We can see that the ZF-CMA is able to cancel ISI as from $17.6 \mathrm{~dB}$ to $-5.4 \mathrm{~dB}$ until stable at $k=700$ and the MMSE-CMA can cancel ISI as from $17.6 \mathrm{~dB}$ to $-9.3 \mathrm{~dB}$ until stable at iterative $k=400$. Consequently, we also point out needed object at $M=4$ and $L=12$. It can be easily seen that the proposed method shows better performance than the conventional method in both regions. We can summarize our proposed approach that the ISI cancellation of MMSE-CMA is better than ZF-CMA by $4 \mathrm{~dB}$ and the convergence rate is faster than ZF-CMA by 100 iterations in case of $M=1$ and $L=2$. In case of $M=2$ and $L=8$, the MMSE-CMA is better than ZF-CMA by $2 \mathrm{~dB}$ and the convergence rate is faster than ZF-CMA by 300 iterations. Also, the ISI cancellation of MMSE-CMA in $M=4$ and $L=12$ is better than $\mathrm{ZF}-\mathrm{CMA}$ by $3 \mathrm{~dB}$ and the convergence rate is faster than ZF-CMA by 200 iterations, respectively. As the results, the proposed method ZF-CMA and MMSE-CMA can be taken to resolve the limitation of the slow convergence rate of the ISI severe at the RFID reader receiver. 


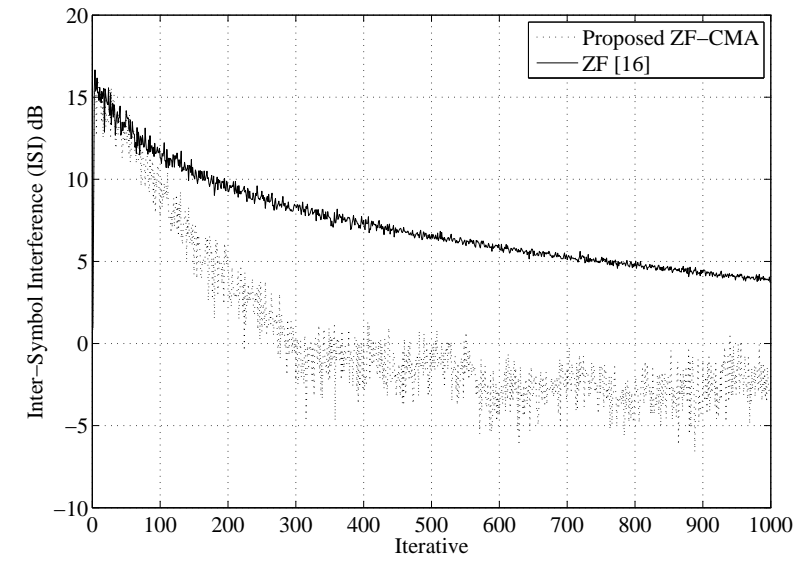

(a) ZF-CMA with $M=1, L=2$

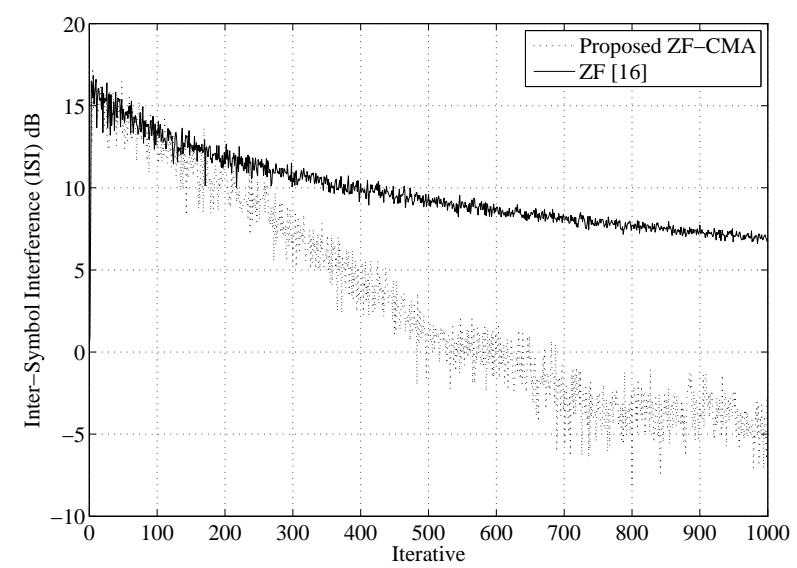

(c) ZF-CMA with $M=2, L=8$

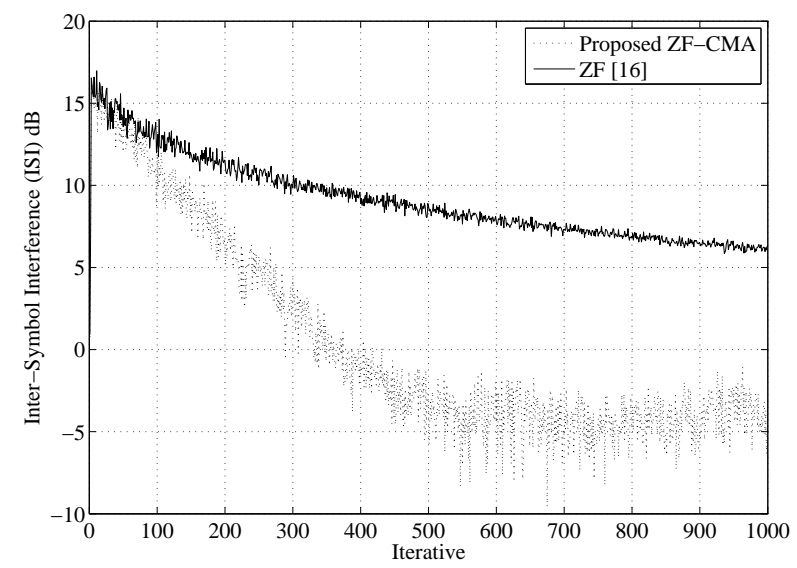

(e) ZF-CMA with $M=4, L=12$

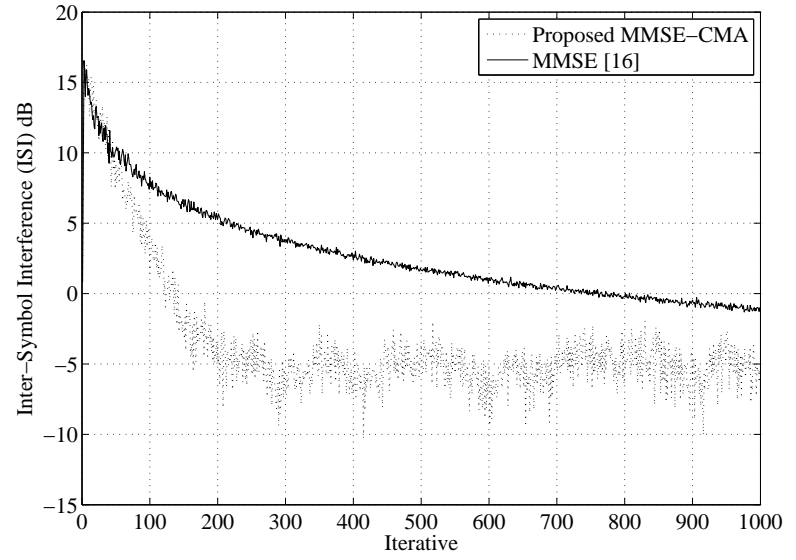

(b) MMSE-CMA with $M=1, L=2$

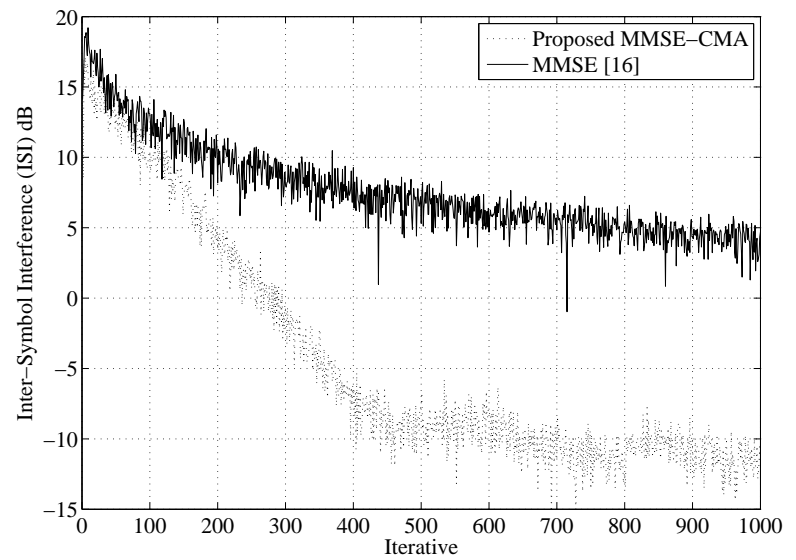

(d) MMSE-CMA with $M=2, L=8$

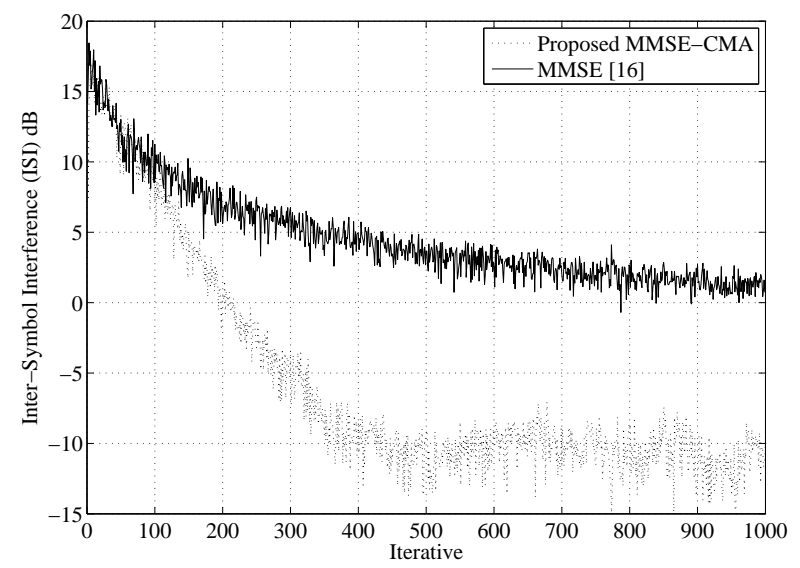

(f) MMSE-CMA with $M=4, L=12$

Fig. 7. Comparison of the ISI cancelled convergence rate between the conventional method and the proposed method. 


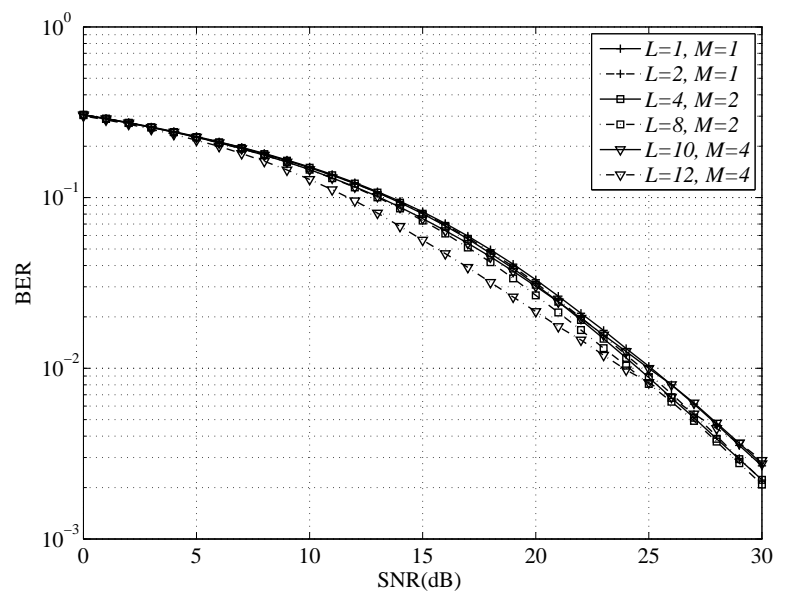

Fig. 8. BER performance via measured channel data with ZFCMA method.

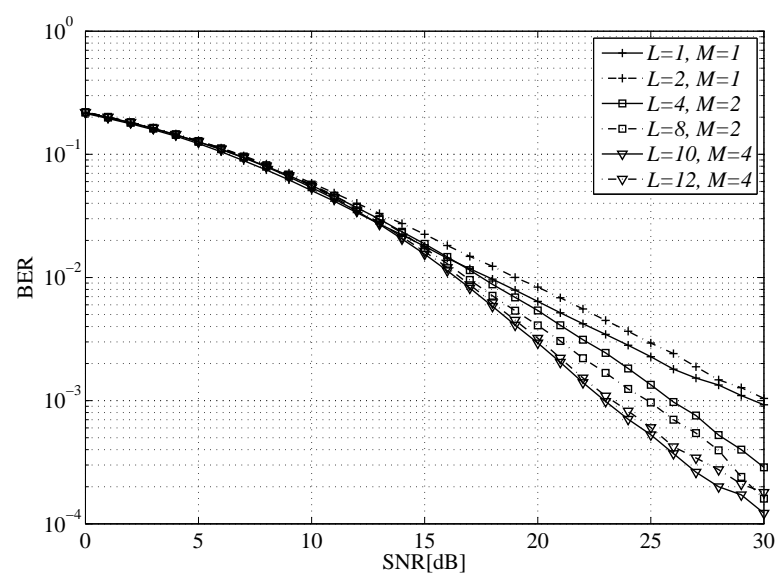

Fig. 9. BER performance via measured channel data with MMSE-CMA method.

Figure 8 and Figure 9 show BER versus SNR performance of the multiple-tag detection with QPSK modulation and $L=1, L=2, L=4, L=8, L=10$, and $L=12$ simulation backscattered through the measured channel data. Figure 8 shows the BER of ZF-CMA method. The performance of ZF-CMA is carried out close to BER $10^{-3}$, where the data rate was simulated as $640 \mathrm{kbps}$ of the passive backscatter. It can be observed that the ZF-CMAs agree with the ISI convergence rates given in Fig. 7, where the convergence rates had not a lower the minimum ISI threshold at $-10 \mathrm{~dB}$. Thus, the BER in ZF-CMA method is still not overcoming the severe ISI perfectly. The BER performance of the MMSECMA method is satisfied by a lower BER $10^{-3}$ as shown in Fig. 9. It is apparent that $M=2$ and $M=4$ has a lower BER of $10^{-3}$, in particular, where SNR $=30 \mathrm{~dB}$. The MMSECMA method is able to cancel ISI better than the ZF-CMA distinguishes that, the SNR is a higher $10 \mathrm{~dB}$ and the convergence speed $k$ is a faster 200 of the iteration.

\section{Conclusion}

The conventional ZF and MMSE methods are not able to rapidly cancel ISI because of the error of post-preamble transmission under the DBC model. In this paper, a new ZF and MMSE based on self-recovering of blind equalizer have been proposed as a solution to the problem of slow convergence rate in ISI cancellation. The convergence rate of ISI cancellation can be resolved by using the cost function to estimate the channel transfer function via second order statistics. Compared with ISI cancelling, the proposed method is better than the conventional method by a percentage of ZF-CMA about 40 percent and the MMSE-CMA is about 60 percent, respectively. Furthermore, we have pointed out the BER performance to guarantee the multiple tag of postprocessing under the assumption of the analysis with maximum likelihood detection. The results confirmed that the proposed method can be accomplished by improving the limit of $L=2 M$, up to 12 tags by the same number of receiving antennas. Nevertheless, an assumption of how to experiment as $L>12$ that will be considered under DBC modeling in the further work.

\section{Acknowledgements}

The authors gratefully acknowledge the comments of the anonymous reviewer and of the associate editor that significantly improved the manuscript. This work was supported by the research fund of the National Broadcasting and Telecommunications Commission (NBTC) Ph.D Program.

\section{References}

[1] BOLIC, M., SIMPLOT-RYL, D., STOJMENOVIC, I. RFID Systems: Research Trends and Challenges. Wiley, 2010.

[2] BOYER, C., ROY, S. Code QAM backscatter modulation for RFID IEEE Transactions on Communications, 2012, vol. 60, no. 7, p. 1925-1934. DOI: 10.1109/TCOMM.2012.051012.110317

[3] ABIDI, A., A. The path to the software-defined radio receiver. IEEE Journal of Solid-State Circuits, 2007, vol. 42, no. 5, p. 954-966. DOI: 10.1109/JSSC.2007.894307

[4] GREY, S., VOM BOGEL, G., GRABMAIER, A. Flexible development and testing environment for implementation of new algorithms in RFID systems. In Proceedings of Fourth International EURASIP Workshop on RFID Technology. Torino (Italy), 2012, p. 35-40. DOI: 10.1109/RFID.2012.9

[5] CANTUCCINI, L., DE DONNO, D., COLELLA, R., RICCIATO, F., TARRICONE, L. A cost-effective SDR platform for performance characterization of RFID tags. IEEE Transaction on Instrumentation and Measurement, 2012, vol. 61, no. 4, p. 903-910. DOI: 10.1109/TIM.2011.2174899

[6] DE DONNO, D., RICCIATO, F., TARRICONE, L. Listening to tags: Uplink RFID measurements with an open-source software-defined radio tool. IEEE Transaction on Instrumentation and Measurement, 2013, vol. 62, no. 2, p. 109-117. DOI: 10.1109/TIM.2012.2212513 
7] YANJUN ZUO. Survivable RFID system: issues, challenges, and techniques. IEEE Transactions on Systems, Man, and Cybernetics, Part C: Applications and Reviews, 2010, vol. 40, no. 4, p. 406-418. DOI: 10.1109/TSMCC.2010.2043949

[8] DUANGSUWAN, S., PROMWONG, S. Performance evaluation of RFID reader with blind filtering equalization for SDR platform. In Proceedings of the $26^{\text {th }}$ International Technical Conference on Circuits/Systems, Computers and Communications. Gyeongju (Korea), 2011, p. 42-45

[9] CHEN HE, WANG, J. Impact of the correlation between forward and backscatter channels on RFID system performance. In Proceedings of IEEE International Conference on Acoustics, Speech and Signal Processing (ICASSP). Prague (Czechia), 2011, p. 3540-3543. DOI: 10.1109/ICASSP.2011.5946242

[10] GRIFFIN, J. D., DURGIN, G. D. Gains for RF tags using multiple antennas. IEEE Transactions on Antennas and Propagations, 2008, vol. 56, no. 2, p. 563-570. DOI: 10.1109/TAP.2007.915423

[11] ZHANG, G., ZHANG, Y., KANG, W. Low-complexity channel estimation for the UHF MIMO-RFID system with optimal training. International Journal of Advancements in Computing Technology, 2012, vol. 4, no. 1, p. 387-394.

[12] HE, C., CHEN, X., WANG, Z. J., SU, W. On the performance of MIMO RFID backscattering channels. EURASIP Journal on Wireless Communications and Networking, 2012, vol. 1, no. 357, p. 1-15. DOI: 10.1186/1687-1499-2012-357

[13] FENG ZHENG, KAISER, T. On the transmit signal design at the reader for RFID MIMO system. In Proceedings of Fourth International EURASIP Workshop on RFID Technology. Torino (Italy), 2012, p. 59-64. DOI: 10.1109/RFID.2012.11

[14] BOYER, C., ROY, S. Space time coding for backscatter RFID. IEEE Transactions on Wireless Communications, 2013, vol. 12, no. 5 , p. 2272-2280. DOI: 10.1109/TWC.2013.031313.120917

[15] MINDIKOGLU, A. F., VAN DER VEEN, A. J. Separation of overlapping RFID signals by antenna arrays. In Proceedings of IEEE International Conference on Acoustics, Speech and Signal Processing (ICASSP). Las Vegas (USA), 2008, p. 2737-2740. DOI: 10.1109/ICASSP.2008.4518215

[16] KAITOVIC, J., LANGWIESER, R., RUPP, M. RFID reader with multi antenna physical layer collision recovery receivers. In Proceedings of IEEE International Conference on RFID-Technologies and Applications. Sitges (Spain), 2011, p. 286-291. DOI: 10.1109/RFIDTA.2011.6068651

[17] DO-YUN KIM, ET AL. Reverse-link interrogation range of a UHF MIMO-RFID system in nakagami- $m$ fading channels. IEEE Transactions on Industrial Electronics, 2010, vol. 57, no. 4, p. 1468-1477. DOI: 10.1109/TIE.2009.2030134

[18] ANGERER, C., LANGWIESER, R., RUPP, M. RFID reader receivers for physical layer collision recovery. IEEE Transactions on Communications, 2010, vol. 58, no. 12, p. 3526-3537. DOI: 10.1109/TCOMM.2010.101910.100004

[19] ANGERER, C., LANGWIESER, R., MAIER, G., RUPP, M. Maximal ratio combining receivers for dual antenna RFID readers. In IEEE MTT-S International Microwave Worskhop on Wireless Sensing, Local Positioning and RFID. Cavtat (Croatia), 24-25 Sept. 2009, p. 1 - 4. DOI: 10.1109/IMWS2.2009.5307888
[20] KAITOVIC, J., SIMKO, M., LANGWIESER, R., RUPP, M. Channel estimation in tag collision scenario. In Proceedings of IEEE International Conference on RFID. Orlando (USA), 2012, p. 74-80. DOI: 10.1109/RFID.2012.6193059

[21] FYHN, K., JACOBSEN, R. M., POPOVSKI, P., SCAGLIONE, A., LARSEN, T. Mutlipacket reception of passive UHF RFID tags: a communication theoretic approach. IEEE Transactions on Signal Processing, 2011, vol. 59, no. 9, p. 4225-4236. DOI: 10.1109/TSP.2011.2159499

[22] BAI YU, ZHANG LIYI, TENG JIANFU. The application of the CMA blind equalization in UHF RFID system. In IET Conference on Wireless, Mobile and Sensor Networks. Shanghai (China), 2007, p. 326-329.

[23] YE LI, LIU, K. J. R. Adaptive blind source separation and equalization for multiple-input/multiple-output systems. IEEE Transaction on Information Theory, 1998, vol. 44, no. 7, p. 2864-2876. DOI: $10.1109 / 18.737518$

[24] TUGCU, E., CAKIR, F., OZEN, A. A new step size control technique for blind and non-blind equalization algorithms. Radioengineering, 2013, vol. 22, no. 1, p. 44-51.

\section{About the Authors ...}

Sarun DUANGSUWAN was born in 1984 in Ranong, Thailand. He received his B.E. degree and M.E. degree in Information Engineering from King Mongkut's Institute of Technology Ladkrabang, in 2009 and 2010 respectively. He has received a scholarship from the National Broadcasting and Telecommunications Commission (NBTC) of Thailand. His research interests are in an RFID systems, channel prediction, and MIMO channels. He is a member of the IEEE and a student member of the IEICE Japan.

Sathaporn PROMWONG was born in 1966 in ChiangMai, Thailand. He received his B.Ind. degree in Electronics and M.E. degree in Electrical Engineering from King Mongkut's Institute of Technology Ladkrabang, in 1994 and 1999, respectively. He received his Ph.D. degree in Communications and Integrated Systems from Tokyo Institute of Technology, Japan in 2008. He is currently working as a lecturer at Department of Telecommunication Engineering, Faculty of Engineering, King Mongkut's Institute of Technology Ladkrabang. He has authored more than 80 publications in journals and proceedings. His research interests are in ultra wideband impulse radio (UWBIR) system, antennas and channel propagation. Dr. Promwong is a member of the ECTI Thailand, IEICE Japan, and IEEE broadcasting. 